\title{
The Effect of Growth and Curvature on Pattern Formation*
}

\author{
R. G. Plaza ${ }^{1}$, F. Sánchez-Garduño ${ }^{2}$, P. Padilla $^{3,4,5}$, R. A. Barrio ${ }^{6}$, \\ and P. K. Maini ${ }^{7}$
}

Received January 13, 2003; revised May 5, 2004

\begin{abstract}
Based on first principles, we derive a general model to describe the spatio-temporal dynamics of two morphogens. The diffusive part of the model incorporates the dynamics, growth and curvature of one- and two-dimensional domains embedded in $\mathbb{R}^{3}$. Our generalized diffusion process includes spatio-temporal varying diffusion coefficients, advection, and dilution terms. We present specific examples by analyzing a third order activator-inhibitor mechanism for the kinetic part. We carry out illustrative numerical simulations on two-dimensional growing domains having different geometries. Comparisons with former results on fixed domains show the crucial role of growth and curvature of pattern selection. Evidence is given that both effects might be biologically relevant in explaining the selection of some observed patterns and in changing or enhancing their stability.
\end{abstract}

KEY WORDS: Pattern formation; curvature; growth; reaction-diffusion.

\footnotetext{
* Dedicated to Professor Shui-Nee Chow on the occasion of his 60th birthday.

${ }^{1}$ Courant Institute of Mathematical Sciences, New York University, 251 Mercer St., New York, NY 10012. E-mail: plaza@cims.nyu.edu

${ }^{2}$ Departamento de Matemáticas, Facultad de ciencias. UNAM, Ciudad Universitaria 04510, México D.F., México. E-mail: faustino@servidor.unam.mx

${ }^{3}$ IIMAS-FENOMEC, UNAM, Apartado Postal 20-726, Admon. No. 20, C.P. 01000, Del. Álvaro Obregón, México, D.F., México

${ }^{4}$ Present address: Mathematical Institute, 24-29 St. Giles. OX1 3LB. Oxford, England. E-mail: padilla@maths.ox.ac.uk; pablo@uxmym1.iimas.unam.mx

${ }^{5}$ To whom correspondence should be. addressed.

${ }^{6}$ Instituto de Física, UNAM, Apartado Postal 20-364, México 01000, D.F., México. E-mail: barrio@anatolia.ifisicacu.unam.mx

${ }^{7}$ Centre for Mathematical Biology, Mathematical Institute, University of Oxford, 24-29 St. Giles, Oxford OX1 3LB. UK. E-mail: maini@maths.ox.ac.uk
} 


\section{INTRODUCTION}

Since the seminal paper by Turing [31], reaction-diffusion models have been proposed to account for pattern formation in a wide variety of biological situations (for a review see [20]). The simplest version of the model consists of two coupled non-linear reaction-diffusion equations describing the spatio-temporal evolution of the concentration of two substances (termed morphogens by Turing). Turing showed that for conditions under which the reaction kinetics admitted a linearly stable spatially uniform steady state, it was possible for diffusion to cause an instability, leading to spatially varying profiles in morphogen concentration. These are the Turing patterns and they arise from the so called diffusion-driven instability. It has been shown that these models exhibit a variety of spatial patterns consistent with those observed in a number of biological systems. However, the identification of morphogens forming patterns via the Turing instability has proved to be elusive so far, although patterns due to a diffusion-driven instability have been observed experimentally in some chemical systems [7,12] and morphogens have been identified in some systems [29].

From a theoretical viewpoint, the hypothesis that spatial patterns in early development arise via a Turing instability has been criticized for a number of reasons. For example, Turing patterns are sensitive to initial conditions and to perturbations in parameter values. However, it has been shown that boundary conditions can have a profound effect on mode selection and robustness of patterning $[2,13]$, at least in the one-dimensional case. It has also been shown, again for the one-dimensional case, that robustness can be enhanced by considering growth of the domain [10].

The original Turing paper did not take into account the effect of domain growth and changes in geometry, yet these processes are a vital part of the development of any organism, leading to changes in patterning that are not simply quantitative, for example, intensity of color, number of stripes, but are also qualitative, for instance, changes in type and spatial location of stripes or spots. One striking example, which has been often cited recently, is the coat pattern of the angelfish Pomacanthus imperator [16].

An important question arises then, as to whether the appearance of some patterns, as well as their stability (instability) can be understood by taking into account the growth of the organism. Several models have been proposed for this and we now briefly review them.

Murray [20] found that changes in spatial scale can produce dramatic changes in the patterns exhibited by the Turing model. In [2] the effect of a growing domain is incorporated by choosing a time-dependent 
scaling factor. In [16] the authors proposed a reaction-diffusion system to describe the changes in the stripe patterns of Pomacanthus as the fish grows. In particular, they showed that the reaction-diffusion system went through a frequency-doubling cascade which preserved the pattern wavelength as the domain grew, consistent with the patterning observed on the fish. Meinhardt et al. have also studied several consequences of growth in pattern formation (see [19] and references therin). In this work a comparison between activator-inhibitor and activator-substrate models is presented.

Varea et al. [32] studied the evolution of Turing patterns in a twodimensional domain with curved boundaries. By imposing specific chemical concentrations on the boundary (simulating a chemical source there) they reproduced some aspects of the developing pigmentation patterns in Pomacanthus. In a subsequent paper, Varea et al. [33] investigated the effect of curvature on pattern selection in a Turing model on a fixed sphere. Their simulations show the emergence of different symmetries on this surface and are reminiscent of skeletal patterns in radiolaria. Turing patterns on a growing sphere were recently investigated numerically by Chaplain et al. [8] in an application to metastasis in a growing tumor. Liaw et al. [17] simulated a Turing model on part of a spherical surface and showed that they could reproduce various patterns that are exhibited on the hard wings of lady beetles.

The case of domain growth being directly controlled by one of the chemicals in the Turing model has been studied on a simple one-dimensional domain [11] and also on complex two-dimensional surfaces [15]. In the latter, the authors show possible applications to morphogenesis of single-celled chlorophyte algae which exhibit branching processes, whorl formation in Acetabularia and repeated dichotomous branching in Micrasterias.

This brief review shows that understanding the effects of growth and geometry on Turing patterns is currently an issue of importance. Therefore, in this paper we consider a general growing two-dimensional surface and investigate, numerically, the effect on the stability of patterns of growth and geometry, particularly the growth rate and the curvature of the domain. Note that the work by Crampin et al. [10] already addresses the effects rate and functional form at least for simple one-dimensional domains.

The main purpose of the paper is to provide a general framework for the study of pattern formation using reaction diffusion equations in which the effects of both growth and geometry are taken into account. The paper is organized as follows. In Section 2 we derive the model for growing and curved one- and two-dimensional domains. We begin 
by considering the diffusive part of the process and then we simply add the nonlinear kinetic interaction terms and write down the equations in non- dimensional form. We then study the special case of isotropic growth and include some remarks on the diffusion-driven instability on fixed twodimensional domains. We conclude by listing some typical geometries and growth functions. In Section 3 we restrict ourselves to a particular model, which includes third order kinetics. Here we carry out the Turing bifurcation analysis for a fixed domain in detail. In Section 4 we present several numerical simulations in order to investigate the differences in the pattern formation process on different surfaces (e.g., the square and the cone) and growing domains. In Section 5 we discuss a number of open problems in this area.

\section{CONSTRUCTION OF THE MODEL}

\subsection{Diffusion in Growing and Curved Domains}

To derive the appropriate form for a system of coupled reaction-diffusion equations on a growing domain, we first parametrize the domain. For the one-dimensional case, let us consider the parameter $s \in[0,1]$ (the spatial parameter) and define the mapping $\psi_{t}$, such that for every time $t \geqslant 0$,

$$
\psi_{t}:[0,1] \longrightarrow \mathbb{R}^{3}, \quad \psi_{t}(s) \equiv X(s, t)=\left(\begin{array}{c}
x(s, t) \\
y(s, t) \\
z(s, t)
\end{array}\right) .
$$

$X(s, t)$ represents a curve in space parameterized by $s$, for each time $t$. This curve, which we denote $\mathcal{C}_{t}$, can be used to represent a one-dimensional spatial domain which grows in time. It will be convenient for later use to introduce at this stage the arc length as a function of $s$ and $t$,

$$
\sigma(s, t)=\int_{0}^{s}\left|X_{s}\left(s^{\prime}, t\right)\right| \mathrm{d} s^{\prime} .
$$

For two-dimensional growth we asume that for every time $t \geqslant 0$, there is a surface $\mathcal{S}_{t}$ parametrized by $(\zeta, \eta) \in \Omega_{0} \subset \mathbb{R}^{2}$ that models the shape and size of the growing domain (the organism). Hence, there is a mapping

$$
\psi_{t}: \Omega_{0} \subset \mathbb{R}^{2} \longrightarrow \mathbb{R}^{3}, \quad \psi_{t}(\zeta, \eta) \equiv X(\zeta, \eta, t)=\left(\begin{array}{c}
x(\zeta, \eta, t) \\
y(\zeta, \eta, t) \\
z(\zeta, \eta, t)
\end{array}\right),
$$

that defines a two-dimensional surface $\mathcal{S}_{t}$ embedded in $\mathbb{R}^{3}$.

Before deriving the equations for the reaction-diffusion system on the growing domains parametrized as above, we make the following simplifying assumptions: 
(a) For every fixed $t \geqslant 0$, the growth function $\psi_{t}: \Omega_{0} \subset \mathbb{R}^{p} \longrightarrow \mathbb{R}^{3}$, for $p=1,2$ is $C^{2}$, and such that if $\Omega_{0}$ is a fixed domain (which could be the whole space $\left.\mathbb{R}^{p}\right)$, then $\Omega_{t} \equiv \psi_{t}\left(\Omega_{0}\right)$ models the geometry of the growing organism. That is $\psi_{t}\left(\Omega_{0}\right)$ is the region occupied by the organism at time $t$ and we also assume it is differentiable with respect to this variable. In addition, we assume that the mapping is continuously differentiable on the parameter $t$.

(b) When $p=1$, Eq. (1) defines a regular curve $\mathcal{C}_{t}$ embedded in $\mathbb{R}^{3}$ for every $t$, that is,

$$
X_{s}(s, t) \neq 0
$$

for all $s \in[0,1]=\Omega_{0}$ and $t \geqslant 0$.

When $p=2$, Eq. (3) defines a regular surface $\mathcal{S}_{t}$ in $\mathbb{R}^{3}$, that is,

$$
X_{\zeta} \times X_{\eta} \neq 0
$$

for all $(\zeta, \eta) \in \Omega_{0}$ and all $t \geqslant 0$.

Now, suppose that $\phi$ denotes the concentration (molecules per unit volume) of a chemical substance, depending on $(X, t) \times \mathbb{R}^{3} \times[0, \infty)$, We make the standard assumption that the morphogen diffuses according to Fick's law, which states that the flux vector $J$ of the substance molecules is proportional to the concentration gradient, i.e.,

$$
J=-D \nabla \phi
$$

where $D \geqslant 0$ is the diffusion coefficient. Consider a region in space $\Omega$, and notice that for a surface element $\mathrm{d} S$ on $\partial \Omega$ with outer unit normal $\mathbf{n},-J \cdot \mathbf{n d} S$ represents the flow of particles out through the element. Then, as usual, by Fick's law we can write

$$
\frac{\mathrm{d}}{\mathrm{d} t} \int_{\Omega} \phi \mathrm{d} X=D \int_{\partial \Omega} \nabla \phi \cdot \mathbf{n} \mathrm{d} S .
$$

In our models, $\phi$ will denote the concentration of the morphogens per unit length or per unit surface. The domain of integration in Eq. (6) is a domain $\Omega$ lying on the curve $\mathcal{C}_{t}$ or on the surface $\mathcal{S}_{t}$, and consequently depends on time. The spatial diffusion takes place in the local variables on the surface or the curve, and we have to take into account both the geometry of the domain and the fact that it varies with time. In other words, the gradient that appears in Fick's law refers to the intrinsic gradient on the curve (surface). 
For the case of two interacting chemicals with concentrations $u$ and $v$, Eq. (6) should be expressed in terms of the concentration vector $\mathbf{w}=$ $(u, v)^{T}$ and the $2 \times 2$ constant matrix of diffusivities $\mathcal{D}=\mathcal{D}_{i j}$,

$$
\frac{\mathrm{d}}{\mathrm{d} t} \int_{\Omega} \mathbf{w} \mathrm{d} X=\mathcal{D} \int_{\partial \Omega} \nabla \mathbf{w} \cdot \mathbf{n} \mathrm{d} S+\int_{\Omega} \mathcal{R}(\mathbf{w}) \mathrm{d} X,
$$

where $\mathcal{R}(\mathbf{w})=(f(u, v), g(u, v))^{T}$ is the reaction kinetics vector. We shall not consider cross diffusion, and the matrix of diffusivities will be regarded as the diagonal matrix $\mathcal{D}=\operatorname{diag}\left(D_{1}, D_{2}\right)$ with $D_{i}$ positive constants. Thus, for the derivation of the diffusive part of the model, it will be sufficient to deal with Eq. (6) for only one substance, and we shall incorporate the kinetic functions later, which will be the only coupling terms in our equations.

\subsubsection{One-Dimension}

Consider a domain which grows in onedimension, parametrized as in Eq. (1). Let $\phi=\phi(X(s, t), t)$ be the morphogen concentration per unit length, and consider a segment of the curve $\mathcal{C}_{t}$, defined by $\Omega(t) \equiv$ $\psi_{t}\left(\left[s_{1}, s_{2}\right], t\right)$, where $\left[s_{1}, s_{2}\right] \subset[0,1]$ and $\psi_{t}$ is the growth function. Making a change of variables we have that

$$
\frac{\mathrm{d}}{\mathrm{d} t} \int_{s_{1}}^{s_{2}} \phi(X(s, t), t) \sigma_{s}(s, t) \mathrm{d} s=D \int_{s_{1}}^{s_{2}} \partial_{s}\left(\nabla_{X} \phi \cdot \frac{\left|X_{s}\right|}{\left|X_{s}\right|}\right) \mathrm{d} s .
$$

Here $\sigma_{s}(s, t)=\left|X_{s}\right|$. We denote $\tilde{\phi}(s, t) \equiv \phi(X(s, t), t)$, and compute the derivatives inside the integrals to obtain

$$
\int_{s_{1}}^{s_{2}}\left(\tilde{\phi}_{t} \sigma_{s}+\tilde{\phi} \sigma_{s t}-D\left(\frac{\tilde{\phi}_{s s}}{\sigma_{s}}-\frac{\sigma_{s s}}{\left(\sigma_{s}\right)^{2}} \tilde{\phi}_{s}\right)\right) \mathrm{d} s=0,
$$

which holds for every interval $\left[s_{1}, s_{2}\right] \subset(0,1)$ and every $t \geqslant 0$. Dropping the tildes for notational convenience we obtain the following equation on the fixed coordinates $s$ and $t$

$$
\phi_{t}=\frac{D}{\left(\sigma_{s}\right)^{2}}\left(\phi_{s s}-\frac{\sigma_{s s}}{\sigma_{s}} \phi_{s}\right)-\frac{\sigma_{s t}}{\sigma_{s}} \phi,
$$

which can also be expressed as

$$
\phi_{t}=\frac{D}{\sigma_{s}} \partial_{s}\left(\frac{1}{\sigma_{s}} \phi_{s}\right)-\partial_{t}\left(\ln \sigma_{s}\right) \phi .
$$


Therefore, for the case of the morphogens, with concentrations $u$ and $v$, respectively, which diffuse on the time-varying curve $\mathcal{C}_{t}$ parametrized by $s$, the corresponding equations are:

$$
\begin{aligned}
& u_{t}=\frac{D_{1}}{\left(\sigma_{s}\right)^{2}}\left(u_{s s}-\frac{\sigma_{s s}}{\sigma_{s}} u_{s}\right)-\frac{\sigma_{s t}}{\sigma_{s}} u \\
& v_{t}=\frac{D_{2}}{\left(\sigma_{s}\right)^{2}}\left(v_{s s}-\frac{\sigma_{s s}}{\sigma_{s}} v_{s}\right)-\frac{\sigma_{s t}}{\sigma_{s}} v .
\end{aligned}
$$

Here $D_{i}>0$ are the constant diffusion coefficients and $\sigma(s, t)$ is determined by the growth function. Note that all the terms on the right-hand side of (9) are space-time dependent. Their physical interpretation is as follows:

$$
\begin{aligned}
\frac{D_{i}}{\left(\sigma_{s}\right)^{2}}(\cdot)_{s s}= & \text { diffusion term, } \quad-D_{i} \frac{\sigma_{s s}}{\left(\sigma_{s}\right)^{3}}(\cdot)_{s}=\text { advection term, and } \\
& -\frac{\sigma_{s t}}{\sigma_{s}}(\cdot)=\text { dilution term, } \quad \text { for } i=1,2 .
\end{aligned}
$$

\subsubsection{Two Dimensions}

Consider the surface $\mathcal{S}_{t}$ embedded in $\mathbb{R}^{3}$ and described in terms of the parameters $(\zeta, \eta) \in \Omega_{0} \subset \mathbb{R}^{2}$ for each value of $t$ by Eq. (3). As usual we define

$$
h_{1}:=\left|X_{\zeta}\right|, \quad h_{2} \equiv\left|X_{\eta}\right|,
$$

and the normal vector is given by $N(\zeta, \eta, t)=X_{\zeta} \times X_{\eta} \neq 0$, since we assume $\mathcal{S}_{t}$ is regular for each $t$. We also have the expression for the metric on the surface, which is given by

$$
\mathrm{d} l^{2}=\mathrm{d} x^{2}+\mathrm{d} y^{2}+\mathrm{d} z^{2}=g_{i j} \mathrm{~d} x_{i} \mathrm{~d} x_{j}
$$

where $x_{1}=\zeta, x_{2}=\eta$, and $g_{i j}=X_{x_{i}} \cdot X_{x_{j}}, i, j=1,2$. We denote

$$
E=\left|X_{\zeta}\right|^{2}=g_{11}, \quad F=X_{\zeta} \cdot X_{\eta}=g_{12}, \quad G=\left|X_{\eta}\right|^{2}=g_{22} .
$$

Here we are assuming that the parametrization $(\zeta, \eta)$ is such that it defines an orthogonal system on $\mathcal{S}_{t}$, that is,

$$
X_{\zeta} \cdot X_{\eta}=0,
$$

for each time $t$. Hence the matrix $G$ of coefficients of the first fundamental form is

$$
G=\left(\begin{array}{ll}
g_{11} & g_{12} \\
g_{21} & g_{22}
\end{array}\right)=\left(\begin{array}{ll}
h_{1}^{2} & 0 \\
0 & h_{2}^{2}
\end{array}\right)
$$


with inverse

$$
G^{-1}=\frac{1}{g_{11} g_{22}}\left(\begin{array}{rr}
g_{22} & -g_{21} \\
-g_{12} & g_{11}
\end{array}\right)=\frac{1}{h_{1}^{2} h_{2}^{2}}=\left(\begin{array}{ll}
h_{2}^{2} & 0 \\
0 & h_{1}^{2}
\end{array}\right) .
$$

Clearly $|N(\zeta, \eta, t)|=h_{1} h_{2}$ at each time $t$. Now, let $\phi$ be the morphogen concentration of a substance on the surface $\mathcal{S}_{t}$, that is, $\phi=\phi(X, t)$ is the number of molecules per unit area at time $t$, and $X \in \mathcal{S}_{t}$. Consider a region $\Omega(t)$ on the surface, where diffusion takes place, and assume $\Omega(t)=$ $\psi_{t}\left(\Omega_{0}\right)$ for some open, bounded domain $\Omega_{0} \in \mathbb{R}^{2}$, with $\partial \Omega_{0}$ smooth. Then the diffusion process for $\phi$ on $\Omega(t)$ is given by

$$
\frac{\mathrm{d}}{\mathrm{d} t} \int_{\Omega(t)} \phi(X, t) \mathrm{d} S_{X}=D \oint_{\partial \Omega(t)} \nabla \phi \cdot \mathbf{n} \mathrm{d} l .
$$

Here $\partial \Omega(t)$ is a regular curve on the surface and $\mathbf{n}$ is the unit vector normal to the curve, which lies on the tangent plane. We suppose that the curve $\partial \Omega(t)$ is the image under the growth function of $\partial \Omega_{0}$, a closed curve in $\mathbb{R}^{2}$, and assume that the former is parametrized by $\left(\zeta_{0}(s), \eta_{0}(s)\right)$. Let $\tau \in \mathbb{R}^{3}$ be the tangent vector to $\partial \Omega(t)$. Therefore, $\tau=\zeta_{0}^{\prime} X_{\zeta}+\eta_{0}^{\prime} X_{\eta}$, with $|\tau|^{2}=h_{1}^{2} \zeta_{0}^{\prime 2}+h_{2}^{2} \eta_{0}^{\prime 2} \neq 0$. In (11), $\mathbf{n}$ lies on the tangent plane, $|\mathbf{n}|=1$ and $\mathbf{n} \cdot \tau=0$; hence $\mathbf{n}=\alpha X_{\eta}+\beta X_{\zeta}$, where

$$
\alpha=-\frac{h_{1}}{h_{2}} \frac{\zeta_{0}^{\prime}}{|\tau|}, \quad \text { and } \quad \beta=\frac{h_{2}}{h_{1}} \frac{\eta_{0}^{\prime}}{|\tau|} .
$$

Changing variables in the line integral along $\partial \Omega(t)$ and applying Stokes theorem on the integral along the curve $\partial \Omega_{0} \subset \mathbb{R}^{2}$ we obtain

$$
\begin{aligned}
\oint_{\partial \Omega(t)} \nabla \phi \cdot \mathbf{n} \mathrm{d} l & =\oint_{\partial \Omega_{0}} \nabla \phi \cdot\left(\alpha X_{\eta}+\beta X_{\zeta}\right)|\tau| \mathrm{d} s \\
& =\oint_{\partial \Omega_{0}}\left(-\frac{h_{1}}{h_{2}} \partial_{\eta} \phi\right) \mathrm{d} \zeta+\left(\frac{h_{2}}{h_{1}} \partial_{\zeta} \phi\right) \mathrm{d} \eta \\
& =\oint_{\Omega_{0}}\left[\left(\frac{h_{2}}{h_{1}} \partial_{\zeta} \phi\right)_{\zeta}+\left(\frac{h_{1}}{h_{2}} \partial_{\eta} \phi\right)_{\phi}\right] \mathrm{d} \zeta \mathrm{d} \eta .
\end{aligned}
$$

The last formula is valid for any time $t \geqslant 0$. Now if we change variables on the left-hand side of Eq. (11) we get

$$
\begin{aligned}
\frac{\mathrm{d}}{\mathrm{d} t} \int_{\Omega(t)} \phi(X, t) \mathrm{d} S_{X} & =\frac{\mathrm{d}}{\mathrm{d} t} \int_{\Omega_{0}} \phi(X(\zeta, \eta, t), t) h_{1} h_{2} \mathrm{~d} \zeta \mathrm{d} \eta \\
& =\int_{\Omega_{0}}\left(\left(\phi_{t}+\nabla \phi \cdot X_{t}\right) h_{1} h_{2}+\phi\left(h_{1} h_{2}\right)_{t}\right) \mathrm{d} \zeta \mathrm{d} \eta .
\end{aligned}
$$


Denote $\tilde{\phi}(\zeta, \eta, t) \equiv \phi(X(\zeta, \eta, t), t)$. Clearly $\tilde{\phi}_{t}=\phi_{t}+\nabla \phi \cdot X_{t}$ and $\tilde{\phi}_{\zeta}=$ $\partial_{\zeta} \phi, \tilde{\phi}_{\eta}=\partial_{\eta} \phi$. Therefore, the molecular diffusion mechanism expressed by Eq. (11) can be written as

$$
\int_{\Omega_{0}} \tilde{\phi}_{t} h_{1} h_{2}+\tilde{\phi}\left(h_{1} h_{2}\right)_{t}-D\left[\left(\frac{h_{2}}{h_{1}} \tilde{\phi}_{\zeta}\right)_{\zeta}+\left(\frac{h_{1}}{h_{2}} \tilde{\phi}_{\eta}\right)_{\eta}\right] \mathrm{d} \zeta \mathrm{d} \eta=0 .
$$

As $\Omega_{0}$ is arbitrary, and dropping the tildes for notational convenience, we obtain

$$
\phi_{t}=D \Delta_{s} \phi-\phi \partial_{t}\left(\ln \left(h_{1} h_{2}\right)\right)
$$

where

$$
\Delta_{s} \phi=\frac{1}{h_{1} h_{2}}\left[\left(\frac{h_{2}}{h_{1}} \phi_{\zeta}\right)_{\zeta}+\left(\frac{h_{1}}{h_{2}} \phi_{\eta}\right)_{\eta}\right],
$$

is the Laplace-Beltrami operator, which is the intrinsic Laplace operator in manifolds. Hence the equations describing the case of two morphogens, with concentration $u$ and $v$, respectively, diffusing on the surface $\mathcal{S}_{t}$ are

$$
\begin{aligned}
& u_{t}=D_{1} \Delta_{s} u-\partial_{t}\left(\ln \left(h_{1} h_{2}\right)\right) u \\
& v_{t}=D_{2} \Delta_{s} v-\partial_{t}\left(\ln \left(h_{1} h_{2}\right)\right) v,
\end{aligned}
$$

where $u=u(\zeta, \eta, t), v=v(\zeta, \eta, t),(\zeta, \eta) \in \Omega_{0}, t \in[0, \infty)$, and $D_{i}>0$ with $i=$ 1,2 are the constant diffusion coefficients.

\subsection{The Full Model}

Recalling the analysis of the previous section, the reaction-diffusion model we wish to study takes the form

For one dimension:

$$
\begin{aligned}
& u_{t}=\frac{D_{1}}{\left(\sigma_{s}\right)^{2}}\left(u_{s s}-\frac{\sigma_{s s}}{\sigma_{s}} u_{s}\right)-\frac{\sigma_{s t}}{\sigma_{s}} u+f(u, v) \\
& v_{t}=\frac{D_{2}}{\left(\sigma_{s}\right)^{2}}\left(v_{s s}-\frac{\sigma_{s s}}{\sigma_{s}} v_{s}\right)-\frac{\sigma_{s t}}{\sigma_{s}} v+g(u, v) .
\end{aligned}
$$

For two dimensions:

$$
\begin{aligned}
u_{t} & =D_{1} \triangle_{s} u-\partial_{t}\left(\ln \left(h_{1} h_{2}\right)\right) u+f(u, v) \\
v_{t} & =D_{2} \triangle_{s} v-\partial_{t}\left(\ln \left(h_{1} h_{2}\right)\right) v+g(u, v) .
\end{aligned}
$$

In both cases the components of the kintetic part, $f(u, v)$ and $g(u, v)$, are given by nonlinear function of the morphogen concentrations. 


\subsubsection{The Nondimensional Form of the Model}

We can carry out the standard procedure to write down the equations in nondimensional form (see [20]) and obtain:

$$
\begin{aligned}
& u_{t}=\frac{d}{\left(\sigma_{s}\right)^{2}}\left(u_{s s}-\frac{\sigma_{s s}}{\sigma_{s}} u_{s}\right)-\frac{\sigma_{s t}}{\sigma_{s}} u+\tilde{\gamma} f(u, v) \\
& v_{t}=\frac{1}{\left(\sigma_{s}\right)^{2}}\left(v_{s s}-\frac{\sigma_{s s}}{\sigma_{s}} v_{s}\right)-\frac{\sigma_{s t}}{\sigma_{s}} v+\tilde{\gamma} g(u, v),
\end{aligned}
$$

for one-dimension. Analogously, in two dimensions

$$
\begin{aligned}
& u_{t}=\frac{d}{h_{1} h_{2}}\left[\left(\frac{h_{2}}{h_{1}} u_{\zeta}\right)_{\zeta}+\left(\frac{h_{1}}{h_{2}} u_{\eta}\right)_{\eta}\right]-\partial_{t}\left(\ln \left(h_{1} h_{2}\right)\right) u+\tilde{\gamma} f(u, v) \\
& v_{t}=\frac{1}{h_{1} h_{2}}\left[\left(\frac{h_{2}}{h_{1}} v_{\zeta}\right)_{\zeta}+\left(\frac{h_{1}}{h_{2}} v_{\eta}\right)_{\eta}\right]-\partial_{t}\left(\ln \left(h_{1} h_{2}\right)\right) v+\tilde{\gamma} g(u, v)
\end{aligned}
$$

where $d=D_{1} / D_{2}$ is the ratio of the diffusion coefficients and the parameter $\tilde{\gamma}$ is similar to the one defined by Arcuri and Murray [2] and by Kondo and Asai in [16]. In their work, the time-dependence of the growing domain was included explicitly in this parameter. Recently Crampin et al. [10] defined a time-dependent $\tilde{\gamma}$ using a growth function for a one-dimensional domain; in their work the coefficients of diffusivity are affected by a factor $1 /(\tilde{\gamma}(t))^{2}$. Our model is a generalization of this particular case. We drop the time dependence of $\tilde{\gamma}$ since it can be expressed via the quantities $\sigma_{s}^{-2}$ and $\left(h_{1} h_{2}\right)^{-1}$ affecting the coefficients of diffusivity, which also include geometrical effects. In what follows, $\tilde{\gamma}$ is the constant appearing above and represents the relative strengths of the reaction terms. Natural questions arise about the effects of the new dilution and advection terms on diffusion-driven instability. In particular we conjecture that the effective space-time varying diffusion coefficients play an important role in both the emergence and the selection of patterns. Linear stability analysis of this system is one of the new open problems to be tackled.

\subsubsection{Isotropic Growth}

We begin with the simplifying hypothesis of isotropic growth. By this, we mean that the growth of the curve or surface takes place in the same proportion in all directions as time increases. In mathematical terms, this means that the growing manifold can be parametrized as follows:

$$
\begin{aligned}
X(s, t) & =\rho(t) X_{0}(s), & & \text { in one dimension, } \\
X(\zeta, \eta, t) & =\rho(t) X_{0}(\zeta, \eta), & & \text { in two dimensions. }
\end{aligned}
$$


This assumption seems natural in physical applications and it simplifies our analysis. Here $X_{0}$ is a fixed two-dimensional (surface) or one- dimensional (curve) manifold. The growth function $\rho(t)$ satisfies our previous regularity assumptions and is constrained to $\rho(0)=1$ and $\dot{\rho}(t)>0$ for all $t$. In this way the effects of curvature and growth can be uncoupled and studied separately. For instance, take the hypothesis of isotropic growth in one dimension. If we denote $:=\mathrm{d} / \mathrm{d} t,^{\prime}:=\mathrm{d} / \mathrm{d} s$, and $\sigma(s)=\int_{0}^{s}\left|X_{0}^{\prime}(\xi)\right| \mathrm{d} \xi$ (the arc length of the "fixed" curve $X_{0}(s)$ ), then Eq. (18) clearly take the form

$$
\begin{aligned}
& u_{t}=\frac{d}{\rho^{2} \sigma^{\prime 2}}\left(u_{s s}-\frac{\sigma^{\prime \prime}}{\sigma^{\prime}} u_{s}\right)-\frac{\dot{\rho}}{\rho} u+\tilde{\gamma} f(u, v) \\
& v_{t}=\frac{1}{\rho^{2} \sigma^{\prime 2}}\left(v_{s s}-\frac{\sigma^{\prime \prime}}{\sigma^{\prime}} v_{s}\right)-\frac{\dot{\rho}}{\rho} v+\tilde{\gamma} g(u, v) .
\end{aligned}
$$

Additionally we have the relations $\sigma^{\prime}=\left|X_{0}^{\prime}\right|, \sigma^{\prime \prime}=\left(X_{0}^{\prime} \cdot X_{0}^{\prime \prime}\right) /\left|X_{0}^{\prime}\right|$. Notice that the arc length satisfies $\dot{\sigma}>0$ for all $s$. If we are considering a growth function such that there is local expansion everywhere, then $\sigma^{\prime} \geqslant 1$. Thus, our equations will be completely determined as long as we provide both the growth function $\rho$ and the "steady" curve $X_{0}(s)$. Although the isotropic growth assumption is not always biologically appropriate, it is still convenient to study as it could provide some insight for pattern selection in the most general case, and could even represent a good model for pattern evolution on animals in which shape changes very little. In addition, it is of intrinsic mathematical interest.

\subsubsection{Diffusion Driven Instability on a Non-growing Two-Dimensional Manifold}

The fact that the diffusion, advection, and dilution terms depend on space and also on time makes it difficult to carry out the standard stability analysis by means of the plane wave decomposition [20]. Turing bifurcation analysis starts with determining the conditions for the appearance of a diffusion-driven instability. For simplicity and for the rest of this section we shall assume the $\rho(t) \equiv 1$, that is, no growth is considered, and consequently we will take into account only geometrical effects. To make our remarks even simpler we shall focus on the two-dimensional model and, in addition, assume that $\mathcal{S}$ is a compact orientable Riemannian manifold embedded in $\mathbb{R}^{3}$. Thus, our linearized equations arround the steady state take the form:

$$
\mathbf{w}_{t}=\mathcal{D} \Delta_{s} \mathbf{w}+\tilde{\gamma} J \mathbf{w},
$$


where $\mathcal{D}$ is the matrix of diffusivities:

$$
\mathcal{D}=\left(\begin{array}{ll}
d & 0 \\
0 & 1
\end{array}\right),
$$

and $\Delta_{s}$ is the Laplace-Beltrami operator in $\mathcal{S}$ defined in Eq. (14). The main difficulty in carry out the appropriate stability analysis is due to the presence of transport coeffiecients, implicit in Laplace-Beltrami operator. We define the linear operator $\mathcal{L}$ as

$$
\mathcal{L}:=\mathcal{D} \triangle_{s}+\tilde{\gamma} J
$$

so that the condition for diffusion driven instability is that the eigenvalues of $\mathcal{L}$ have positive real part for certain wave numbers (eigenvalues) associated with $\triangle_{S}$ on $\mathcal{S}$. We start by observing the following standard result (see, for example [23], pp. 703-704]): The spectrum of the Laplace-Beltrami operator $-\Delta_{s} f=-g^{j i} \nabla_{i} \nabla_{j} f$ on a compact orientable Riemannian manifold $\mathcal{S}$, and denoted by $\sigma\left(-\triangle_{S}\right)$, has the following properties:

(1) $\sigma\left(-\triangle_{S}\right)$ has no finite accumulation point and lies on the positive real line, that is, it forms a discrete sequence $0=v_{0}<v_{1}<\cdots v_{k}<$ $\ldots$ where $v_{k} \rightarrow+\infty$ as $k \rightarrow \infty$.

(2) For each $v \in \sigma\left(-\triangle_{s}\right)$, the associated eigenspace is finite-dimensional, and

(3) The eigenspaces corresponding to different eigenvalues are orthogonal.

Hence, let us denote the set of scalar eigenfunctions of $\Delta_{s}$ on $\mathcal{S}$ as $w_{k}$, such that

$$
\Delta_{s} w_{k}=-k^{2} w_{k} .
$$

Based on the analysis by Chaplain et al. [8] for the particular case of the unit sphere, we are able to prove a more general result for certain manifolds, given in the following lemma.

Lemma 1. Let $\mathcal{S}$ be a compact orientable Riemannian manifold embedded in $\mathbb{R}^{3}$. A complex number $\lambda \in \mathbb{C}$ is in the spectrum of $\mathcal{L}$ if and only if there exists $k \in \mathbb{R}$ and a scalar eigenfunction $w_{k}$ of the Laplace-Beltrami operator on $\mathcal{S},\left(\triangle_{s} w_{k}=-k^{2} w_{k}\right)$, such that

$$
\operatorname{det}\left(-\mathcal{D} k^{2}+\tilde{\gamma} J-\lambda I\right)=0 .
$$

Proof. For the "if" part, let us assume that the determinant is 0 for some $k \in \mathbb{R}$. Then take $\mathbf{w}_{0} \in \operatorname{ker}\left(-\mathcal{D} k^{2}+\tilde{\gamma} J-\lambda I\right) \subset \mathbb{C}^{2}$. Therefore,

$$
(\mathcal{L}-\lambda I) w_{k} \mathbf{w}_{0}=w_{k}\left(-\mathcal{D} k^{2}+\tilde{\gamma} J-\lambda I\right) \mathbf{w}_{0}=0 .
$$


The "only if" part follows by supposing that the determinant is different from 0 for all $k \in \mathbb{R}$ and some $\lambda \in \mathbb{C}$. Consider $\mathcal{L}$ as an operator in $L^{2}\left(\mathcal{S}, \mathbb{C}^{2}\right) . \mathcal{L}$ is conjugate to its Fourier transform $\hat{\mathcal{L}}$, with $(\hat{\mathcal{L}}-\lambda I) \hat{\mathbf{w}}(k)=$ $\left(-\mathcal{D} k^{2}+\tilde{\gamma} J-\lambda J\right) \hat{\mathbf{w}}(k)$. The right hand side of the last equation is invertible for all $k$, so we can write $(\mathcal{L}-\lambda I)^{-1}$ as the convolution with the Fourier transform of $\left(-\mathcal{D} k^{2}+\tilde{\gamma} J-\lambda I\right)^{-1}$, and hence $\lambda$ is in the resolvent.

The spatially uniform steady state will become unstable for suitable spatially varying disturbances if the solution to the spectral problem $\mathcal{L} \mathbf{w}=$ $\lambda \mathbf{w}$ satisfies $\operatorname{Re} \lambda>0$ for some $k \neq 0$. By the standard stability theory in [20], Eq. (25) allows us to arrive at the conditions for diffusion driven instability. The excited modes will be associated with the eigenfunctions of $\Delta_{s}$ on $\mathcal{S}$, just as was stated by Chaplain et al. for the particular case of the sphere. We point out that formally, the conditions are the same as for the standard Turing instability. However, we emphasize that the geometrical effects on the actual selection of modes are contained in the operator $\mathcal{L}$, via the selection of $k$, the wave number.

We start by examining the conditions for pattern emergence for the case of isotropic growth. This allows us to isolate geometrical effects from growth effects. The isotropic nondimensional model has the following form:

$$
\begin{aligned}
& u_{t}=\frac{d}{\rho(t)^{2}} L u-\frac{\dot{\rho}}{\rho} u+\tilde{\gamma} f(u, v) \\
& v_{t}=\frac{1}{\rho(t)^{2}} L v-\frac{\dot{\rho}}{\rho} v+\tilde{\gamma} g(u, v),
\end{aligned}
$$

where $(x, t) \in \mathbb{R}^{p} \times \mathbb{R}^{+}$. For $p=2, L$ is a second-order differential spatial operator which is simply the Laplace-Beltrami operator defined before while for $p=1$ it is given by $L u=\left(u_{s s}-\left(\sigma^{\prime \prime} / \sigma^{\prime}\right) u_{s}\right) / \sigma^{\prime}(s)^{2}$. Recall that $d$ is the ratio of diffusivities and $\tilde{\gamma}$ is the scale factor introduced in Section 2.2.1. To complete the mathematical problem we must add certain boundary conditions, such as Dirichlet or zero flux type (for studying self-organization of patterns) or periodic boundary conditions (for finite domains excluding effects on boundaries).

\subsection{Examples}

We now consider some concrete examples to illustrate the application of the above ideas:

\subsubsection{Typical Growth Functions}

Typical growth functions $\rho(t)$ that we will consider are: 
(a) No growth: Here $\rho(t) \equiv 1$. for all $t$. This case reduces to Turing's model and serves as the "control" case to enable us to identify the effects of domain curvature and also how pattern changes when we do indeed impose growth.

(b) Linear growth: $\rho(t)=1+b t$, where $b>0$. Although such a growth function is probably biologically implausible, its relative simplicity justifies its consideration as a base case.

(c) Exponential growth: $\rho=\exp (k t)$, where $k>0$. This is a reasonable growth model for the initial phases of growth of certain tissues.

(d) Logistic or saturated growth: In this case the growth function is given by

$$
\rho(t)=\frac{\exp (k t)}{1+\frac{1}{m}(\exp (k t)-1)},
$$

where $k>0$ and $m>1$. Notice that $\rho(0)=1$ and $\rho \rightarrow m>1$ as $t \rightarrow \infty$.

In this case, growth is initially approximately exponential before finally saturating. This is, in a phenomenological sense, a biologically reasonable growth function.

\subsubsection{Typical Geometries in One-Dimension}

Let us constrain ourselves to the isotropic growth assumption. In one dimension the general model is given by Eq. (22). Hence, let us consider the simplest domains:

(a) Straight line: After the non dimensionalization in the previous section, we can consider the "planar" domain defined by

$$
X_{0}(s)=\left(\begin{array}{l}
s \\
0 \\
0
\end{array}\right), \quad \text { for } s \in[0,1] .
$$

Here $\sigma^{\prime}=\left|X_{0}^{\prime}\right|=1$ and $X_{0}^{\prime \prime}=0$. Thus there are no transport terms due to curvature or other geometrical properties. Therefore, Eq. (22) takes the form:

$$
u_{t}=\frac{d}{\rho(t)^{2}} u_{s s}-\frac{\dot{\rho}}{\rho} u+\tilde{\gamma} f
$$

and similarly for the inhibitor $v$. This is the special case studied by Crampin et al. [10] (compare with Eq. (20) in [10], p. 1101). Substitution of the different growth functions leads to different 
systems of equations. Neumann boundary conditions at $s=0$ and $s=1$ complete the mathematical problem.

(b) Ellipse: Consider the ellipse given by

$$
X_{0}(s)=\left(\begin{array}{c}
p \cos s \\
q \sin s \\
0
\end{array}\right), \quad \text { for } s \in[0,2 \pi]
$$

where $p$ and $q$ are positive numbers. We can compute $X_{0}^{\prime} \cdot X_{0}^{\prime \prime}$ and $\left|X_{0}^{\prime}\right|^{2}$ to arrive at

$$
\begin{aligned}
u_{t}= & \frac{\mathrm{d}}{\rho(t)^{2}\left(p^{2} \sin ^{2} s+q^{2} \cos ^{2} s\right)}\left(u_{s s}-\frac{\left(p^{2}-q^{2}\right) \sin (2 s)}{2\left(p^{2} \sin ^{2} s+q^{2} \cos ^{2} s\right)} u_{s}\right) \\
& -\frac{\dot{\rho}}{\rho} u+\tilde{\gamma} f .
\end{aligned}
$$

This example is helpful in the study of geometrical effects. The choice of the growth function and periodic boundary conditions completes the mathematical problem to be studied. The case of a circular closed ring corresponds to the case $p=q=1$, and since $X_{0}^{\prime} \cdot X_{0}^{\prime \prime}=0$ and $\left|X_{0}^{\prime}\right|=1$, we notice that we arrive at the same system of Eq. (28) as in the straight line case. Here case, of course, the necessary boundary conditions are periodic rather than the usual zero flux case for the straight line domain.

(c) Parabola: Consider the open curved domain defined by

$$
X_{0}(s)=\left(\begin{array}{c}
s \\
a s^{2} \\
0
\end{array}\right), \quad \text { for } s \in \mathbb{R}
$$

Computing $\sigma^{\prime}(s)=\sqrt{1+4 a s^{2}}, \sigma^{\prime \prime}(s)=4 a^{2} s / \sigma^{\prime}$ we obatin

$$
u_{t}=\frac{d}{\rho(t)^{2}\left(1+4 a^{2} s^{2}\right)}\left(u_{s s}-\frac{4 a^{2} s}{1+4 a^{2} s^{2}} u_{s}\right)-\frac{\dot{\rho}}{\rho} u+\tilde{\gamma} f .
$$

Any other one-dimensional domain could be treated in a similar fashion.

\subsubsection{Typical Geometries in Two-Dimensions}

We now consider a selection of two-dimensional manifolds, which serve as model systems for the study of the effects of curvature and shape. We present three specific cases: a planar domain, a disk and a cone. 
(a) Planar domain: The simplest case is the one of a planar domain which grows isotropically in time. In this case the model for the domain is simply

$$
X(\zeta, \eta, t) \equiv \rho(t)\left(\begin{array}{l}
\zeta \\
\eta \\
0
\end{array}\right),
$$

and clearly the system of equations reduces to the typical reaction-diffusion model in a plane with a time-varying diffusion coefficient due to growth:

$$
u_{t}=\frac{\mathrm{d}}{\rho^{2}}\left(u_{\zeta \zeta}+u_{\eta \eta}\right)-\frac{2 \dot{\rho}}{\rho} u+\tilde{\gamma} f .
$$

When there is no growth, we obtain Turing's original model in two-dimensions for a planar fixed domain.

(b) Growing sphere: Consider the isotropically growing sphere with radius $\rho$ :

$$
X(\zeta, \eta, t) \equiv \rho(t)\left(\begin{array}{c}
\sin \eta \cos \zeta \\
\sin \eta \sin \zeta \\
\cos \eta
\end{array}\right),
$$

where $\zeta \in[0,2 \pi], \eta \in[0, \pi]$. We have

$$
h_{1}^{2}=\left|X_{\zeta}\right|^{2}=\rho^{2} \sin ^{2} \eta, \quad h_{2}^{2}=\left|X_{\eta}\right|^{2}=\rho^{2}, \quad \frac{h_{1}}{h_{2}}=\sin \eta .
$$

Consequently, Eq. (19) take the form:

$$
u_{t}=\frac{d}{\rho^{2}}\left(u_{\eta \eta}+\frac{1}{\sin ^{2} \eta} u_{\zeta \zeta}-\frac{\cos \eta}{\sin \eta} u_{n}\right)-\frac{2 \dot{\rho}}{\rho} u+\tilde{\gamma} f .
$$

On account of $\sin ^{2} \eta \geqslant 0$ for $\eta \in[0, \pi]$. we have singular effective diffusion coefficients.at the boundaries These equations have been used for the calculations in [33], with $\dot{\rho}=0$.

(c) Growing cone: To illustrate the effects of curvature we shall consider the following domain:

$$
X(\zeta, \eta, t) \equiv \rho(t)\left(\begin{array}{c}
\zeta \cos \eta \\
\zeta \sin \eta \\
a \zeta
\end{array}\right),
$$


where $\zeta \in(0, \infty), \eta \in[0,2 \pi]$ and $a$ is the aspect ratio of the cone. Therefore,

$$
h_{1}^{2}=\left|X_{\zeta}\right|^{2}=\left(1+a^{2}\right) \rho^{2}, \quad h_{2}^{2}=\left|X_{\eta}\right|^{2}=\zeta^{2} \rho^{2}, \quad \frac{h_{1}}{h_{2}}=\frac{\sqrt{\left(1+a^{2}\right)}}{\zeta} .
$$

Hence, the equation for one morphogen takes the form

$$
u_{t}=\frac{d}{\left(1+a^{2}\right)[\rho(t)]^{2}}\left(u_{\zeta \zeta}+\frac{\left(1+a^{2}\right)}{\zeta^{2}} u_{\eta \eta}+\frac{1}{\zeta} u_{\zeta}\right)-\frac{2 \dot{\rho}}{\rho} u+\tilde{\gamma} f
$$

\section{SPECIFIC MODEL}

For the remainder of this paper we focus on reaction kinetics of the model introduced in [4]. This is

$$
f(u, v) \equiv \alpha u\left(1-r_{1} v^{2}\right)+v\left(1-r_{2} u\right), \quad g(u, v) \equiv \beta v\left(1+\frac{\alpha r_{1}}{\beta} u v\right)+u\left(\gamma+r_{2} v\right) .
$$

This particular kinetics was proposed because it captures many of the mechanisms crucial in pattern generation and selection. By suitable choices of the parameters $\alpha$ and $\beta$, the uniform steady state $(0,0)$ can be driven unstable by diffusion. Furthermore, it is known that quadratic interactions select spot patterns, while third order terms favor the selection of stripes [14,21]. In Eq. (39), the parameters $r_{1}$ and $r_{2}$ measure the strength of the third and second order interactions, respectively.

\subsection{Linear Stability Analysis for Fixed Domains}

The stationary states $\left(u^{*}, v^{*}\right)$ of the system of Eq. (39) are the intersection points of the nullclines

$$
\alpha u\left(1-r_{1} v^{2}\right)+v\left(1-r_{2} u\right)=0 \text { and } \beta v+\alpha r_{1} u v^{2}+u\left(\gamma+r_{2} v\right)=0 .
$$

It is easy to show that $\left(u^{*}, v^{*}\right)$ must satisfy the equation

$$
v=-\frac{(\alpha+\gamma)}{(1+\beta)} u, \quad \text { with } \beta \neq-1 .
$$

Observe that, apart of the origin $(u=v=0)$, in principle the system (39) has two more equilibria. The abcissae of these points are the real roots of the equation

$$
u_{0}^{2}+b u_{0}+c=0 \text {, }
$$


where

$$
\varepsilon:=\frac{(\alpha+\gamma)}{(1+\beta)}, \quad u=u_{0} / \varepsilon, \quad b=-\frac{r_{2}}{r_{1} \alpha}, \quad \text { and } \quad c=\frac{(\gamma-\alpha \beta)}{\alpha r_{1}(1+\beta)} .
$$

In order to keep the analysis as simple as possible we impose the condition $\alpha=-\gamma$ which, for finite values of $u$, implies $v^{*}=0$. Hence, from the equations of the nullclines, we have $\gamma u^{*}=0$, and, if we consider $\gamma \neq 0$, then we have that the only finite equilibrium of the system (39) is $(0,0)$. We are currently studying the general case without this condition, and the results will be presented elsewhere [17, in preparation].

The linear approximation of the kinetics in Eq. (39) around the equilibrium $(0,0)$ is

$$
\begin{aligned}
& \dot{u}=-\gamma u+v \\
& \dot{v}=\gamma u+\beta v,
\end{aligned}
$$

from which we have that the trace and determinant of the Jacobian matrix are: $\operatorname{tr} J[f, g]_{(0,0)}=(\beta-\gamma)$ and $\operatorname{det} J[f, g]_{(0,0)}=-\gamma(1+\beta)$, respectively. The eigenvalues of the Jacobian matrix $J[f, g]_{(0,0)}$ are

$$
\lambda_{1}, \lambda_{2}=\frac{(\beta-\gamma) \pm \sqrt{(\beta-\gamma)^{2}+4 \gamma(\beta+1)}}{2} .
$$

By using the linear approximation (42), one readily derives the usual conditions under which $(0,0)$ can exhibit a diffusiondriven instability [20,4] for fixed domains. By choosing the appropriate parameters in the Turing space the instability mechanism can be triggered. Now, we are going to seek the parameter values compatible with the above conditions. Let us consider the possibilities, depending on the sign of $\gamma$ :

Case 1: $\gamma>0$. In order to satisfy all the conditions one must choose $\beta$ such that $\beta<\gamma, \beta<-1$ and $\beta>\left(D_{2} / D_{1}\right) \gamma>0$. There is no value of $\beta$ satisfying all these restrictions.

Case 2: $\gamma<-1$. In this case there are no values of $\beta$ for which all conditions are satisfied,

Case 3: $-1<\gamma<0$. One can verify that the values of $\beta$ for which the above conditions in $[4,20]$ hold are: $-1<\left(D_{2} / D_{1}\right) \gamma<\beta<\gamma$.

In what follows we shall fix the value of $\gamma=-\alpha=-0.899$. Therefore, $\beta$ has to be negative and larger than -1 . The previous analysis allows us to choose suitable parameters in Turing space for which $(0,0)$ is linearly unstable in the presence of diffusion, and the subsequent dynamics is governed by the full nonlinear terms, which we investigate numerically. 


\section{NUMERICAL CALCULATIONS IN TWO-DIMENSIONS}

Given that Crampin et al. [10] have carried out a considerable number of numerical simulations for one-dimensional growing domains by considering different growth functions (exponential, logistic) we restrict our calculations to several simple two-dimensional growing manifolds. There have been previous numerical calculations of patterns forming in static domains of different sizes to simulate growth [32]. This situation would correspond to a very slow growing regime $(t=\epsilon \tau$, with $\epsilon \sim 0)$ when the diffusion terms are not affected by growth. In effect, in Eq. (22) $\dot{\rho}$ is negligible and an expansion of $1 / \rho^{2}$ up to linear order in $\epsilon \tau$ is constant, resulting in the usual non-growing equations and justifying the previous calculations in very slow growing domains. In order to show simultaneously the effects of geometry and of growth on pattern selection, we present simple numerical calculations in two-dimensional manifolds assuming a fast reaction rate $(\epsilon=1)$ and isotropic growth. For simplicity, in all the calculations shown here we choose a linear growth function $\rho(t)=1+b t$, where $b>0$ and at $t=0$ it is assumed that the size is normalized. In all the calculations, the Turing system is solved by a simple Euler method, and therefore time is discretized, that is $t=m \Delta t$, where $m$ is an integer.

\subsection{Growing Planar Domain}

The simplest case is the one of a planar domain which grows isotropically in time. In this case the discretized version of the model (see [4]), in which a finite variation of the concentrations $\Delta u$ and $\Delta v$ is calculated for a discrete increment of time $\Delta t$, can be written as

$$
\begin{aligned}
& \Delta u=\left[\delta d \nabla^{2} u-\delta^{\prime} u+\alpha u\left(1-r_{1} v^{2}\right)+v\left(1-r_{2} u\right)\right] \Delta t \\
& \Delta v=\left[\delta \nabla^{2} v-\delta^{\prime} v+\beta v\left(1+\frac{\alpha r_{1}}{\beta} u v\right)-u\left(\alpha-r_{2} v\right)\right] \Delta t .
\end{aligned}
$$

where $\delta=\delta_{0} / \rho^{2}$ and $\delta^{\prime}=2 \dot{\rho} / \rho$. Here $\delta$ conveniently sets the spatial scale and $\delta_{0}$ gives the initial size of the domain. In these equations $u$ and $v$ represent $N \times M$ matrices defined in a grid, and the Laplacian is discretized in the grid with lattice sites denoted by $(i, j)$. The form is

$$
\left.\nabla^{2} u\right|_{(i, j)}=\left(\begin{array}{l}
{\left[a_{r}(i, j) u(i+1, j)-u(i, j)\right]+} \\
{\left[a_{l}(i, j) u(i-1, j)-u(i, j)\right]+} \\
{\left[a_{u}(i, j) u(i, j+1)-u(i, j)\right]+} \\
{\left[a_{d}(i, j) u(i, j-1)-u(i, j)\right]}
\end{array}\right),
$$

where the matrix elements of $a_{r}, a_{l}, a_{u}$ and $a_{d}$ are unity except at the boundary, where they are set equal to zero when the lattice site has no 
$\operatorname{right}(r), \operatorname{left}(l), \operatorname{up}(u)$ or down $(d)$ neighbor, respectively. This ensures zero flux boundary conditions, and it is equivalent to setting $\hat{n} \cdot \nabla u=0$ at the boundary with normal vector $\hat{n}$. The initial values for the matrices are taken as random everywhere.

In Fig. 1, typical results are shown for $u$, which is defined by a gray scale in the plane. We only show one morphogen pattern in all figures, since we verified that the concentration of $v$ is qualitatively the same as $u$ with a phase shift of $\pi$. The stability of the numerical scheme used in our simulations was analysed out in [1]. There the authors used a finite-differences scheme to solve the space discretization of their system ensuring that the discrete intervals used in the simulations are correct. We observe that the value $b=5 \times 10^{-4}$ was small enough to allow a fair convergence of the patterns.

Observe that the square symmetry appears when the domain is very small. This effect of the domain size was already observed elsewhere [3]. As the domain grows, new spots emerge in the diagonals of the lattice. This occurs until the domain is large enough and existing spots split. This is in agreement with former similar calculations using the Schnakenberg model $[9,18]$. The final result is that for large enough domains one obtains the commonly found hexagonal lattice. This was verified by performing a calculation taking the last pattern as initial condition and stopping the growth to allow the pattern to converge in a bigger lattice. The calculation was not carried out to larger sizes because the spots become of the order of the finite spatial grid, and their resolution is not accurate.

\subsection{Growing Sphere}

There have been various numerical calculations in the past that deal with a spherical domain. In particular, some of us have performed static calculations on spheres of different sizes [33]. The simulations by Chaplain et al. [8] cover the case of dynamical growth. Nevertheless we must point out that there are two main differences in their model and ours: they do not consider the dilution term and they use the Schnakenberg kinetic term. We do not include simulations on the sphere, since we intend to make a more detailed comparison in a future publication.

\subsection{Growing Cone}

Here we consider the lateral surface of a cone in which we cut off its base. The cone itself stands on a horizontal plane. The variables $(\eta, \zeta)$ are defined as $(i, j)$ in a $M \times N$ grid. Now, the discrete equations for our model read exactly as in (43), except that the differential operator $\nabla^{2} u$ has 


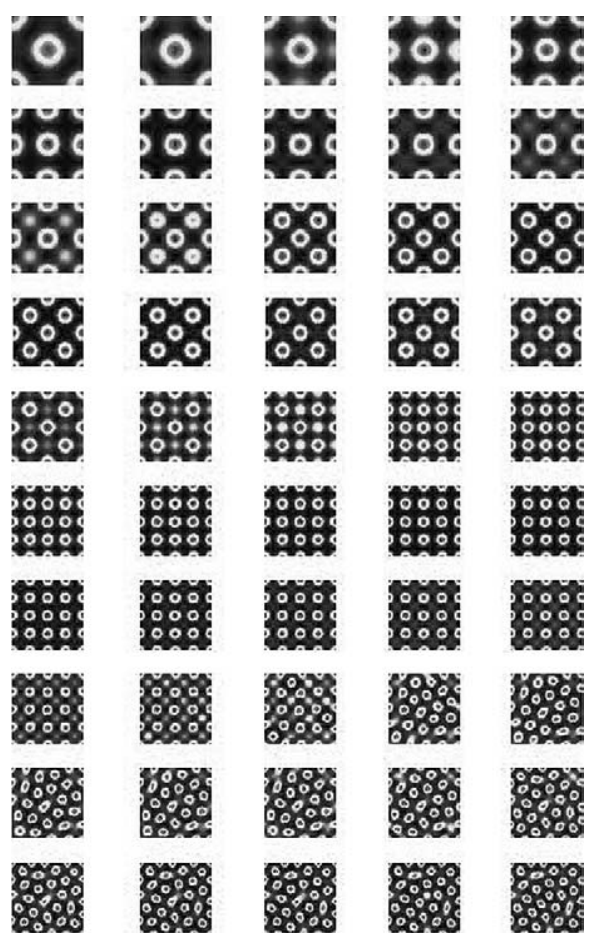

Figure 1. Numerical calculation in a growing square domain of $N=M=48$. The concentration of $u$ is shown in a gray scale. Each one of the 50 pictures is a snap taken every 5000 time steps of $\Delta t=0.02$. The initial $\delta_{0}=11$, and the growth factor is $b=5 \times 10^{-4}$. Therefore, for the last picture in the bottom right $\delta=0.2956$ and the final size is $\rho_{f}=6.1$. The parameters for the system were: $d=0.516, \alpha=0.899, \beta=-0.91, r_{1}=0.5$, and $r_{2}=0.4$, in order to give a post pattern.

to be discretized according to Eq. (24) following the methodology carried out in [1] and replaced by

$$
\begin{aligned}
\mathcal{L} u(i, j)= & \mathrm{d}[u(i, j+1)+u(i, j-1)-2 u(i, j)] \\
& +\frac{\left(1+a^{2}\right)}{j^{2}}[u(i+1, j)+u(i-1, j)-2 u(i, j)] \\
& +\frac{1}{2 j}[u(i, j+1)-u(i, j-1)],
\end{aligned}
$$

and the same for $v(i, j)$. The effective diffusion coefficient is now

$$
\delta=\frac{\delta_{0}}{\left(1+a^{2}\right) \rho(t)^{2}},
$$


where $a$ is the aspect ratio of the cone. For $a=0$ one has a disk, for $a=1$ the cone has a height of the same size as the radius of its base, and for $a>1$ the cone is acute. Care has to be taken to perform the calculation away from $j=0$ to avoid singularities, this means that the cone is really truncated at its apex, by removing a very small piece. The boundary conditions have to be periodic in the angular variable $\eta$, and zero flux at the bottom of the cone $(j=M)$. At the minimum value of $j$ the boundary condition is that the functions at a given point $i$ have the same value as that at the corresponding point $i+\pi$, so continuity at the singular point is fulfilled. One of the things we noticed here is that the selected pattern on the surface of the cone strongly depends on its aspect ratio. Thus, it is worth presenting several typical cases.

\subsubsection{The Disk}

When $a=0$, we have a disk whose radius grows continuously. Barrio et al. [3] already considered a static disk embedded in a square. They incorporate the growth of the disk not as part of an inner dynamics but by simply carrying out the numerical simulations at different increasing radius. We used their static calculations to check that the previous results are reproduced.

In the present case, when the growth rate is comparable with the reaction times, the patterns have not enough time to become stable, and one obtains a series of transient patterns whose symmetry and form changes continuously. However, the series of centrosymmetric patterns observed in the static (or slow growth) regime are still recognizable [3].

In Fig. 2 we show a series of patterns of $u$ obtained numerically in a grid of $M=48$ and $N=24$ starting with a size given by $\delta_{0}=0.8$. Observe that for a very small size one obtains only a central spot, and as the domain grows centered patterns with various symmetries, notably five, emerge. The spot pattern is not as regular as in the static calculations, because there is no time for a given structure to settle. However, several centrosymmetric patterns appear as the disk grows, notably with five-, six- and seven-fold symmetry, respectively, in agreement with previous static calculations [1].

\subsubsection{Symmetric Cone}

We now consider the case where the radius of the base is equal to the height. With no loss of generality, we took $R=a=1$. Numerical results are illustrated in Fig. 3 where we show the distribution of the morphogen $u$ on the surface of the cone. We include the top views at the right of each pattern to facilitate the appreciation of the symmetry. 


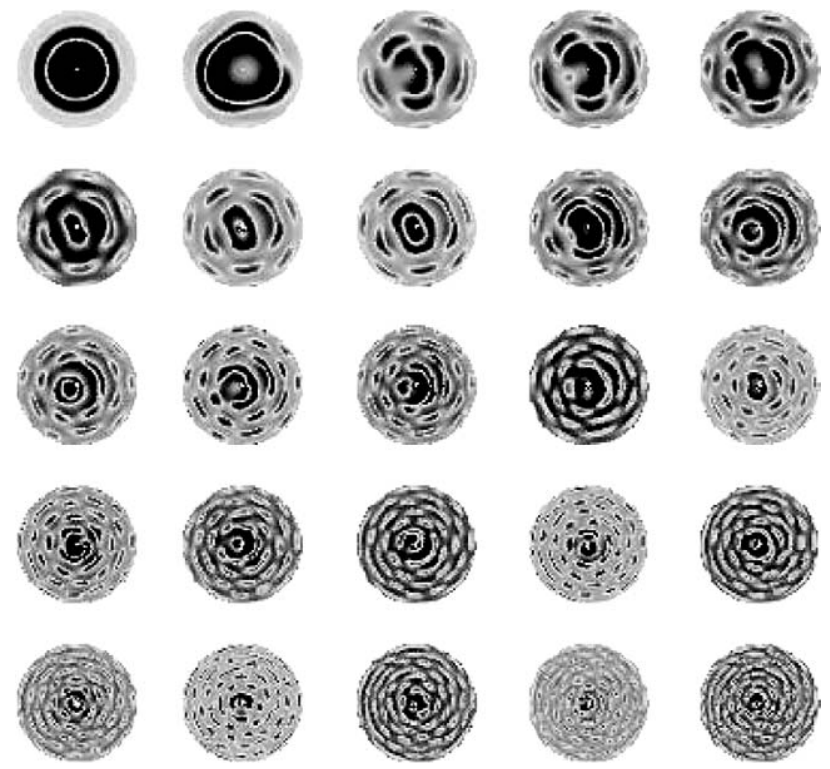

Figure 2. Numerical calculation showing the concentration of $u$ in a growing disk obtained for the equations for the cone with $a=0$. Each picture is taken every 10000 time steps of $\Delta t=0.01$. The growing factor is again $b=3.5 \times 10^{-4}$. The parameters for the system were kept as in the former figure.

Observe that as the cone grows several centrosymmetric patterns arise, notably with three and five fold. When the cone is large these symmetries are lost and lines of spots start appearing.

\subsubsection{Acute Cone}

Here we show that peculiar patterns arise when the aspect ratio of the cone changes. For instance, Fig. 4 shows a calculation on a cone with aspect ratio $a=2$. The parameters are exactly as in Fig. 3. One remarkable feature revealed by the simulations is the evolution from rings on the tip to spots.

When the cone is bigger the annular stripes disappear and the spots align in a sort of helicoidal fashion that resembles pine cones. This is a beautiful example of a pattern modified by the curvature of the surface.

To illustrate this point better we show a numerical calculation using $a=3$ in Fig. 5. Here we have also changed some of the parameters: the initial size is given by $\delta_{0}=1.8$ (smaller starting size than before), the growth rate was somewhat faster, $b=5 \times 10^{-4}$, and each panel shows 

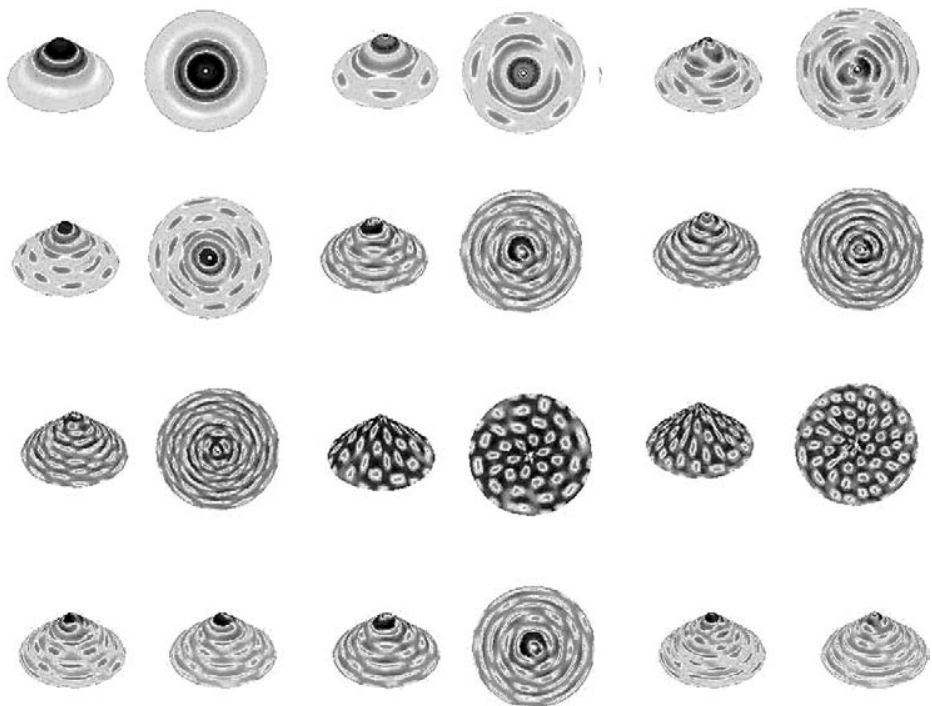

Figure 3. Series of patterns obtained on a cone of aspect ratio one $(a=1)$ with zero-flux boundary conditions at the bottom. The values of the parameters are exactly as in the previous figure except that each snap is taken every 100000 time steps. To the right of each cone there is a top view to facilitate the recognition of the pattern angular symmetry.
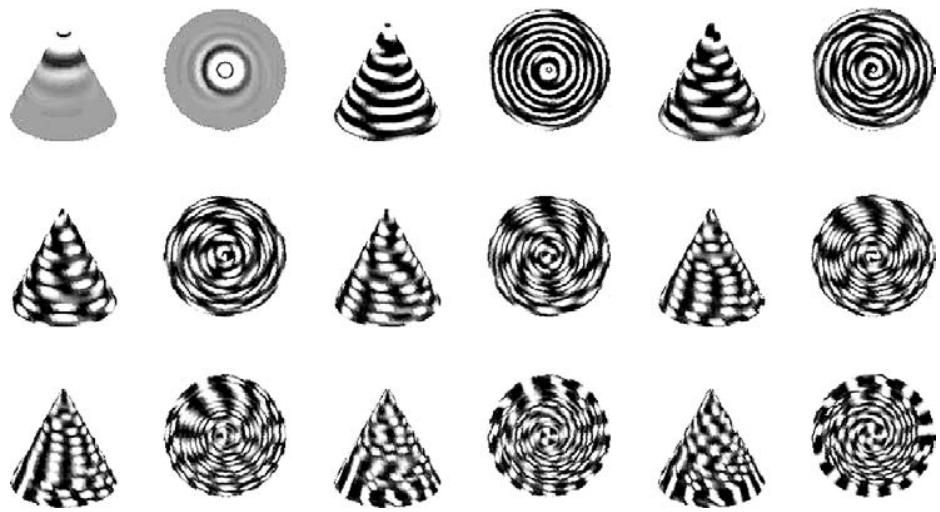

Figure 4. Numerical calculation on a growing cone with $a=2$. Each picture is taken every 100000 time steps of $\Delta t=0.01$. The parameters for the system were kept as in the former figure. Observe the alignment of the spots and the appearance of vertical stripes for large cones. 


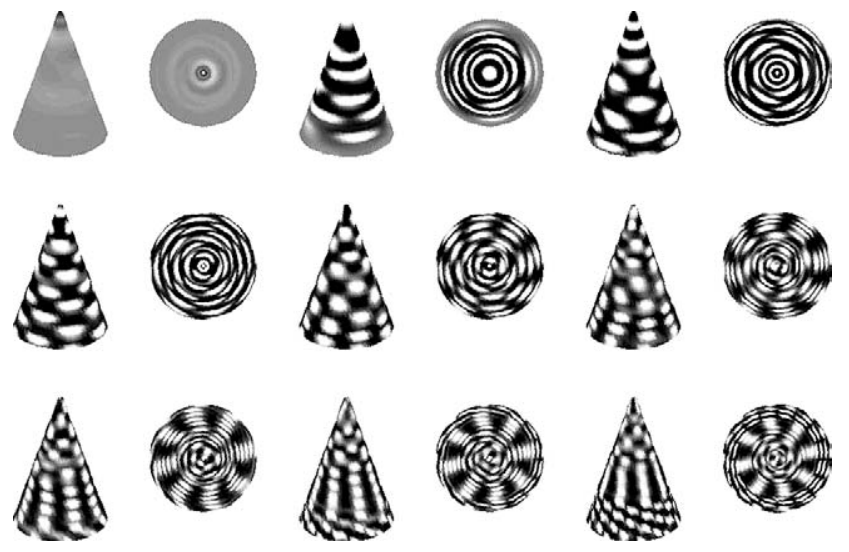

Figure 5. Numerical calculation on a growing cone with $a=3$. The parameters for the system are given in the text. Observe that vertical stripes form more easily in this case, compared to Fig. 4.

results every 100000 time steps, thus the final linear scale was 3.15 and the final $\delta=0.1814$.

These patterns resemble very much the ones observed on the tails of reptilians, like lizards, where it is seen that a pattern of aligned spots and stripes coexist. The important feature that these calculations show is that the interplay of curvature and growth rate of the domain dictate the final selection of the pattern, without having to invoke a variation of the diffusion rate or of the non-linear parameters $\left(r_{1}\right.$ and $\left.r_{2}\right)$. They also show that transient patterns may be important when considering pattern formation in a growing animal.

\subsubsection{Static Cone}

For comparison purposes, we carry out some numerical calculations on non-growing cones until they converged to a stable pattern. We took two cases corresponding to cones of different size and the same aspect ratio from the transient patterns already shown.

In Fig. 6 we show the comparison of a pattern obtained by growing the cone from $\delta=1.8$ to a final size of $\delta=0.2363$ using a grid of $N=24$ and $M=48$ and a growth rate $b=5 \times 10^{-4}$ and we compare it with the pattern obtained in a cone of constant size $\delta=0.2363$. Observe that the alignment of spots caused by the domain growth is lost in the static pattern. The same effect is even more noticeable in a similar calculation on a cone of larger size, shown in Fig. 7. This shows that transient processes 

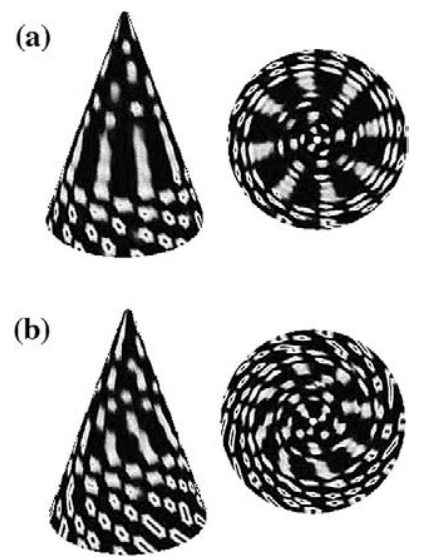

Figure 6. (a) Final steady state of the pattern shown in the bottom right of Fig. 5. (b) Pattern obtained in a cone of constant size equal to the one in (a). Observe that the alignment of spots is lost in the static calculation.

(a)

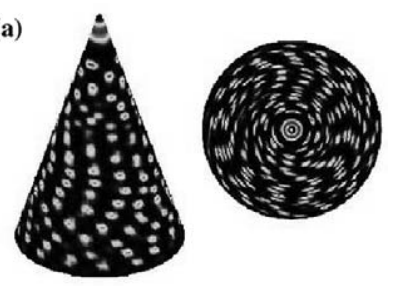

(b)

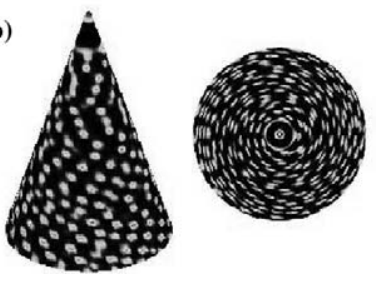

Figure 7. As Fig. 6, except that the final size of the cone is larger, and the grid used was $N=48$ and $M=96$. The time step was adjusted to $\Delta t=0.002$ to assure convergence.

can play an important role in selecting the pattern of a growing animal, since the stable pattern has no time to appear. 


\section{CONCLUSIONS AND DISCUSSION}

We have presented a general theoretical framework to investigate the role of curvature and growth of one- and two-dimensional domains in pattern formation and selection via the Turing instability. The theory can be applied to other space-time dynamics not presented here. The formal bifurcation analysis (local and global) for our full reactive-diffusive models which take into account the dynamics of the one- or two-dimensional domain embedded in $\mathbb{R}^{3}$ is an important challenge from both theoretical and applied points of view. In fact, the corresponding Turing bifurcation analysis for these growing domains still as an unsolved problem which we are presently pursiung. (We are presently carrying out some analysis of these matters.)We presented some specific examples with explicit forms of the reaction equations, restricting ourselves to the case of isotropic growth. In this case, our equations allow a separate examination of the geometrical spatial effects and of the ones due to growth.

It is well documented (see [6]) that there are individuals which grow in a proportional manner. This means that all macroscopic characteristic lengths (length, width, height) keep the same proportion as the individual develops. The result of this is that one adult looks like an amplification in three dimensions of its juvenile stage. Equivalently, its contour at later stages is a homogeneous magnification of that at earlier stages. This is isometric growth which occurs in some fishes like 'Cichlasoma (Nandopsis) urophthalmus' (Günther 1862). Meanwhile there are other organisms which grow in such a way that they do not preserve the proportions between their different linear typical dimensions. This is allometric growth (see [25]). Pattern selection could crucially depend on the two types of growth. For example, Crampin et al. [11] considered a one-dimensional growing domain which grows in a different manner in certain subdomains. One of the challenges in this area is the incorporation of allometric growth. It is also interesting to point out that seasonal effects on the growth of an organism can be incorporated into the model.

We also presented several numerical calculations in growing domains to illustrate the new features that are due to growth. We have restricted our examples to a linear growing function, in order to keep uniformity and for the sake of simplicity and comparison, but it is clear that other simple functions could be easily implemented in a numerical program, according to the specific application of interest. The important result rendered by these calculations is that new patterns can be robustly selected due to the effect of either curvature and/or growth, which would be unstable otherwise. 
In particular, the calculations on the cone show a peculiar alignment of spots, closely resembling the actual array found in reptilian tails and in pine acorns. We are convinced that transient patterns during domain growth are important in simulating living organisms. The analysis of pattern formation, selection, and stabilization on domains with geometries resembling the actual shape of animal bodies, is a difficult task and this provides challenging work for the future.

\section{ACKNOWLEDGMENTS}

This research (PP and FS-G) was partially supported by Consejo Nacional de Ciencia y Tecnología (CONACYT)-México, under the project 34203-E. F.S.-G. and P.K.M. want to thank the Isaac Newton Institute for Mathematical Sciences of the University of Cambridge, for the hospitality during the final preparation of this paper. P.K.M. acknowledges support from a Royal Society Leverhulme Trust Fellowship. R.G.P. was partially supported by DGAPA-UNAM, México, through a Fellowship for Doctoral Studies, and by Dirección de Relaciones Internacionales, SEP, México, Grant 204-6-07106. R.A.B. thank the Department of Theoretical Physics, University of Oxford for the hospitality where part of the numerical simulations of this work were carried out.

\section{REFERENCES}

1. Aragón, J. L., Torres, M., Gil, D., Barrio, R. A., and Maini, P. K. (2002). Turing patterns with pentagonal symmetry. Phys. Rev. E 65(3), 051913-1-9.

2. Arcuri, P., and Murray, J. D. (1986) Pattern sensitivity to boundary and initial conditions in reaction-diffusion models. J. Math. Biol. 24, 141-165.

3. Barrio, R. A., Maini, P. K., Aragón, J. L., and Torres, M. (2002). Size-dependent symmetry breaking in models for morphogenesis. Phys. D 168-169, 61-72.

4. Barrio, R. A., Varea, C., Aragón, J. L., and Maini, P. K. (1999). A two-dimensional numerical study of spatial pattern formation in interacting Turing systems. Bull. Math. Biol. 61, 483-505.

5. Benson, D. L., Maini, P. K., and Sherratt, J. A. (1998). Unravelling the Turing bifurcation using spatially varying diffusion coefficients. J. Math. Biol. 37, 381-417.

6. von Bertalanffy, L., (1973). General System Theory: Foundations, Developments, Applications, Harmondsworth-Penguim, New York.

7. Castets, V., Dulos, E., Boissonade, J., and De Kepper, P. (1990). Experimental evidence of a sustained Turing-type equilibrium chemical pattern. Phys. Rev. Lett. 64, 2953-2956.

8. Chaplain, M. A. J., Ganesh, M., and Graham, I. G. (2001). Spatio-temporal pattern formation on spherical surfaces: Numerical simulation and application to solid tumour growth. J. Math. Biol. 42, 387-423.

9. Crampin E. J. (2000). Reaction-diffusion patterns in growing domains, $\mathrm{PhD}$ thesis, University of Oxford.

10. Crampin, E. J., Gaffney, E. A., and Maini, P. K. (1999). Reaction and diffusion growing domains: Scenarios for robust pattern formation. Bull. Math. Biol. 61, 1093-1120. 
11. Crampin, E. J., Hackborn, W. W., and Maini, P. K. (2002). Pattern formation in reactiondiffusion models with nonuniform domain growth. Bull. Math. Biol. 64, 746-769.

12. De Kepper, P., Castets, V., Dulos, E., and Boissonade, J. (1991). Turing-type chemical patterns in the chlorite-iodide-malonic acid reaction. Phys. D 49, 161-169.

13. Dillon, R., Maini, P. K., and Othmer, H. G. (1994). Pattern formation in generlised Turing systems: I. Steady-state patterns in systems with mixed boundary conditions. J. Math. Biol. 32, 345-393.

14. Ermentrout, G. B. (1991). Stripes or spots? Nonlinear effects in bifurcation of reactiondiffusion equations on the square. Proc. Roy. Soc. London Ser. A 434, 413-417.

15. Harrison, L. G., Wehner, S., and Holloway, D. M. (2002). Complex morphogenesis of surfaces: Theory and experiment on coupling of reaction-diffusion patterning to growth. Faraday Discuss. 120, 277-293.

16. Kondo, S., and Asai, R. (1995). A reaction-diffusion wave on the skin of the marine angelfish Pomacanthus. Nature 376, 765-768.

17. Liaw, S. S., Yang, C. C., and Liu, R. T. (2001). Turing model for patterns of lady beetles. Phys Rev. E. 64, 041909.

18. Madzvamuse, A. (2001). A numerical approach to the study of spatial pattern formation, D.Phil. thesis, University of Oxford.

19. Meinhardt, H., Koch, A.-J., and Bernasconi, G. (1998). Models of pattern formation applied to plant development. In Barabe, D., and Jean, R. V. (eds.), Symmetry in Plants, World Scientific Publishing.

20. Murray, J. D. (1993). Mathematical Biology, Springer.

21. Nagorcka, B. N., and Mooney, J. R. (1992). From stripes to spots: Prepatterns which can be produced in the skin by a reaction-diffusion system. IMA J. Math. Appl. Med. Biol. 9, 249-267.

22. Ni, W.-M. (1998). Diffusion, cross-diffusion, and their spike-layer steady states. Notices AMS, Jan., 9-18.

23. Okubo, T. (1987). Differential Geometry. Dekker.

24. Perumpanani. A. J., Sherratt, J. A., and Maini, P. K. (1995). Phase differences in reactiondiffusion-advection systems and applications to morphogenesis. IMA J. Appl. Math. 55, 19-33.

25. Reiss, Michael (1989). The Allometry of Growth and Reroduction, Cambridge University Press.

26. Rubinstein, J. and Wolansky, G. (1994). Instability results for reaction diffusion equations over surfaces of revolution. J. Math. Anal. Appl. 187, 485-487.

27. Schnakenberg, J. (1979). Simple chemical reaction systems with limit cycle behaviour. J. Theor. Biol. 81, 389-400.

28. Sherratt, J. A. (1995). Turing bifurcation with temporally varying diffusion coefficients. J. Math. Biol. 33, 295-308.

29. Srinivasan, S., Rashka, K. E., and Bier, E. (2002). Creation of a Sog morphogen gradient in the Drosophila embryo. Dev. Cell 2, 91-101.

30. Timm, U., and Okubo, A. (1992). Diffusion-driven instability in a predator-prey system with timevarying diffusivities. J. Math. Biol. 30, 307-320.

31. Turing, A. M. (1952). The chemical basis of morphogenesis, Philos. Trans. R. Soc. Lond. B. 237, 37-72.

32. Varea, C., Aragón, J. L., and Barrio, R. A. (1997). Confined Turing patterns in growing systems. Phys. Rev. E. 36, 1250-1253.

33. Varea, C., Aragón, J. L., and Barrio, R. (1999). Turing patterns on a sphere. Phys. Rev. E 60, 4588-4592. 